\title{
Synchronous multiple primary non-small cell lung cancer or intrapulmonary metastasis from an index lesion: a dilemma-case report
}

\author{
Soumya Guha ${ }^{1}$, Roberto Cascone ${ }^{2}$, Annalisa Carlucci ${ }^{2}$, Panagiota Kitsanta $^{3}$, Laura Socci ${ }^{1}$ \\ ${ }^{1}$ Department of Cardiothoracic Surgery, Northern General Hospital, Sheffield, UK; ${ }^{2}$ Thoracic Surgery Unit, University of Campania Luigi \\ Vanvitelli, Naples, Italy; ${ }^{3}$ Department of Histopathology, Sheffield Teaching Hospital NHS Foundation Trust, Sheffield, UK \\ Correspondence to: Soumya Guha. Department of Cardiothoracic Surgery, Northern General Hospital, Sheffield S5 7AU, UK. \\ Email: surya_blue@yahoo.co.uk.
}

\begin{abstract}
Lung cancer is considered one of the major causes of cancer-related mortality. Our case report wishes to highlight the confusion one may have when faced with a lesion that could be a synchronous lesion or metastasis from an index lesion, and its management including whether re-do VATS is a good option. We report our case of a 68 years old male who came to our attention in March 2018 with history of weight loss and repeated chest infections. He was smoking until he was reviewed in our clinic, with a 40pack year history. PET scan revealed an intense uptake of left lower nodule of size $3.5 \mathrm{~cm}$ (cT2aN0M0). He underwent uniportal VATS left basal segmentectomy and lymphadenectomy with the histopathology confirming a squamous cell carcinoma (SCC) (pT2aN0M0R0). During his follow-up, a new left upper lobe lesion was noticed in February 2019. PET scan showed increased uptake in the lesion and CT guided biopsy showed poorly differentiated SCC (cT1cN0M0). The patient underwent uniportal VATS left upper trisegmentectomy and lymphadenectomy with histopathology revealing the presence of two different lesions this time around: the larger one being a SCC with a staging of pT1cN0M0R0 and the smaller being a basaloid type of SCC with a staging of pT1aN0M0. In our opinion, the three cancerous nodules the patient developed represented multiple primary tumours arising on a background of field change rather than intrapulmonary metastasis from an index lesion (differentiating this was important from management point of view). Moreover, we managed to safely perform a redo surgery via uniportal VATS for the same sided lung lesion. This is feasible as long as the patient has an adequate cardiorespiratory function. We wish to take this opportunity to add to the literature our experience with this case of multiple primary lung cancer (MPLC).
\end{abstract}

Keywords: Multiple primary lung cancer (MPLC); non-small cell lung cancer (NSCLC); VATS re-do surgery; field cancerization; case report

Received: 10 October 2019; Accepted: 05 November 2019; Published: 19 December 2019.

doi: $10.21037 /$ ccts.2019.11.04

View this article at: http://dx.doi.org/10.21037/ccts.2019.11.04

\section{Introduction}

Lung cancer is considered one of the major causes of cancer-related mortality. The risk of developing a second primary lung cancer in all patients successfully treated for a first non-small cell lung cancer (NSCLC), is estimated to be about $1-2 \%$ per patient per year (1); this is explained by a growing hypothesis, known as field cancerization. Even today, the differential diagnosis between a second primary lesion, a recurrence or a metastasis remains difficult. However, this is crucial for the correct staging and management as well as for the patient prognosis. Our case report wishes to highlight the confusion one may have when faced with a lesion that could be a synchronous lesion or metastasis from an index lesion, and its management including whether re-do VATS is a good option. This particular case motivated us to delve more into the 

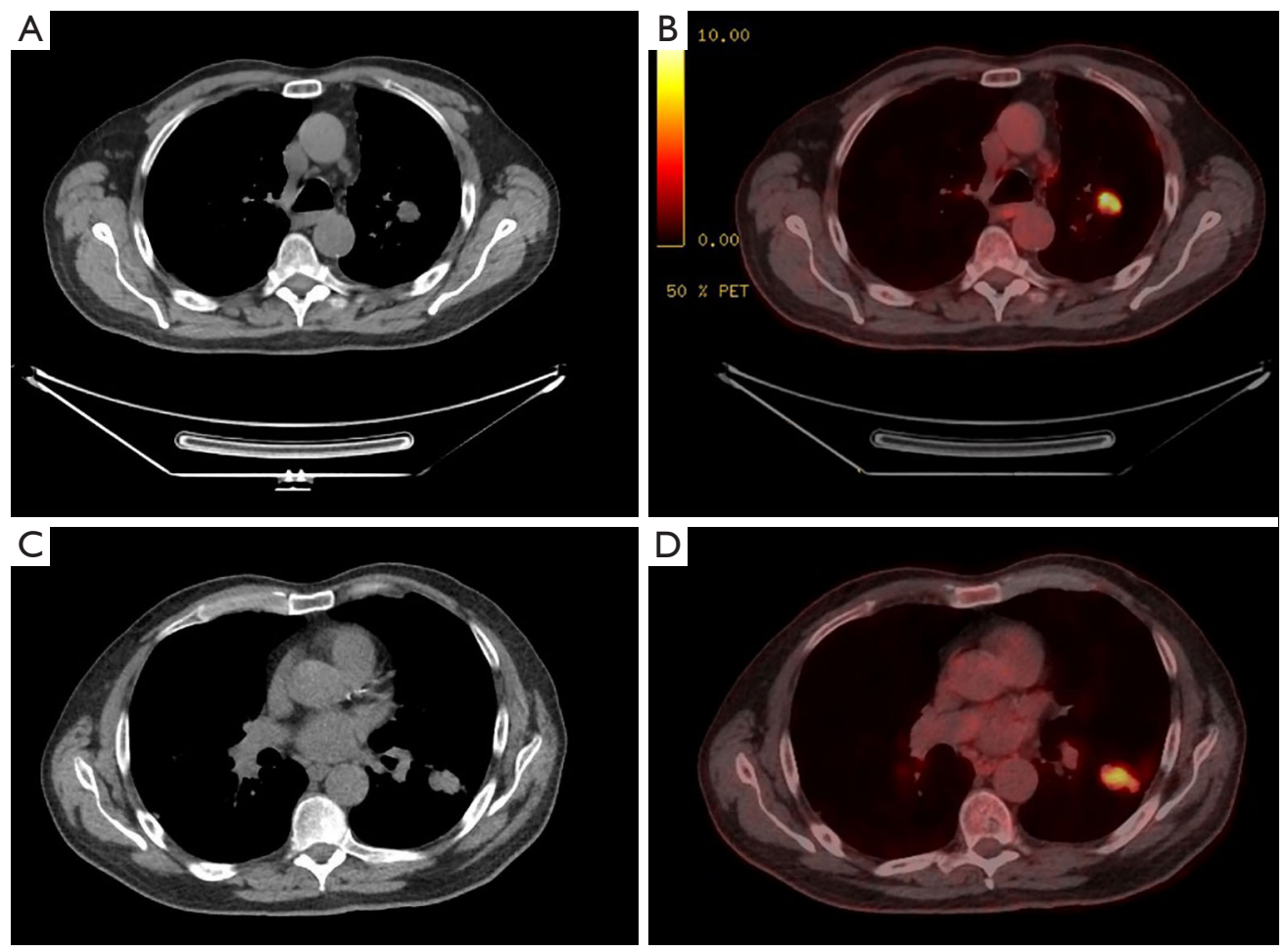

Figure 1 Upper section showing CT and PET of left upper lobe lesion [2019], lower section showing CT and PET of left lower lobe lesion [2018].

background on multiple primary lung cancer (MPLC) and share our findings.

The case report is presented in accordance with the CARE Guideline.

\section{Case presentation}

A 68-year-old male presented with weight loss and repeated chest infections. He was a smoker with a 40-pack year history and no significant family history. His performance status was 1 . His PFTs were preserved with VO2 $\max$ of $20.7 \mathrm{~mL} / \mathrm{kg} / \mathrm{min}$. His PET scan showed a $3.5 \mathrm{~cm}$ nodule with intense uptake in the left lower lobe (staging cT2aN0M0). He underwent left VATS basal segmentectomy and lymphadenectomy on 20/03/2018 with a pathological staging of pT2aN0M0R0 of squamous cell carcinoma (SCC). During his follow-up a new left upper lobe lesion was noticed in February 2019. PET scan showed increased uptake in the lesion (Figure 1) and CT guided biopsy showed poorly differentiated SCC (staging of cT1cN0M0). He was offered both options: a second surgery or stereotactic ablation radiotherapy (SABR).
The patient opted for surgery. The surgical team agreed that the lesion looked resectable and patient's cardiorespiratory reserve was adequate to warrant a surgery. On 16/04/2019 left uniportal VATS upper trisegmentectomy and lymphadenectomy was performed. Histopathology revealed multiple primary tumours. Tumour 1 was SCC (pT1cN0M0R0). Tumour 2 at a distance of $5 \mathrm{~mm}$ from the first tumour, was a basaloid type of SCC (pT1aN0M0R0). We discussed the findings with the pathology team who were of the opinion that the presence of two tumours with different morphologies in the specimen and the history of SCC in 2018 (Figure 2), when taken into consideration, indicate that the specimen represents multiple primary tumours arising on a background of field change rather than intrapulmonary metastasis from an index lesion. This decision and a discussion at the Lung multi-disciplinary meet guided our follow up. Post-operatively the patient is recovering well with a strict follow-up routine involving clinical review and chest $\mathrm{X}$-ray every 3 months for 2 years and then 6 monthly up to 5 years, CT scan if anything unusual, as per the International consensus statement and BTS guidelines. The patient is adhering to this schedule 

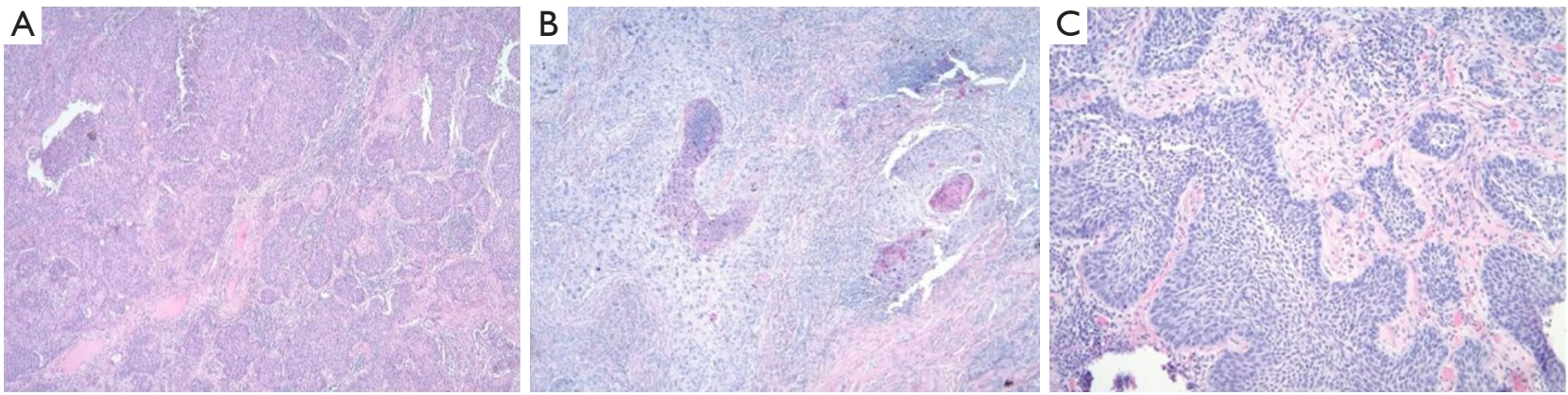

Figure 2 Histopathology slides of the excised tumours. (A) The first excised tumour is moderately differentiated squamous cell carcinoma with foci of keratinisation (HE stain, $\times 10$ ); (B) the subsequent excision a year later: the first tumour is keratinising squamous cell carcinoma (HE stain, $\times 20$ ); (C) the subsequent excision a year later: The second tumour is a basaloid squamous carcinoma (HE stain, $\times 20)$.

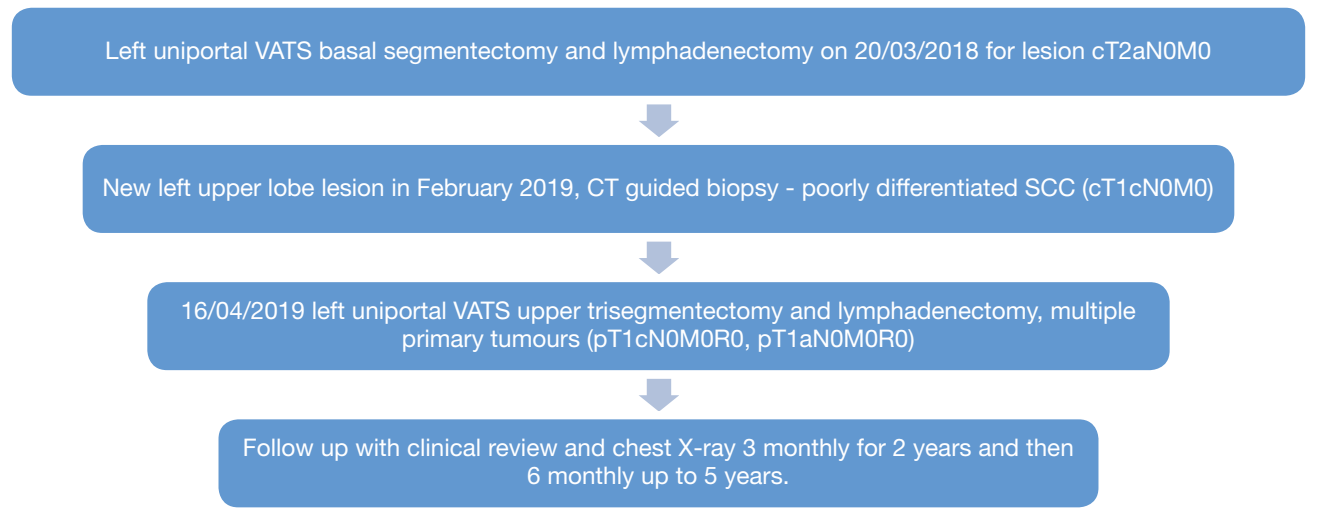

Figure 3 Time-line.

and has been in for the first 2 follow-ups at time of writing the paper (Figure 3).

\section{Discussion}

To date, there are no commonly accepted guidelines for diagnosis and treatment for MPLC. The concept of MPLC was first introduced in 1924 (2) and the criteria for the diagnosis of MPLC stated by Martini and Melamed (3) still serve as the main pillar. These were improved by analysis of molecular genetic characteristics introduced by the American College of Chest Physicians Lung Cancer Guidelines and by Antakli et al. (4). According to these criteria, we define as synchronous a second tumour detected and resected simultaneously, that is distinct and separate from the primary, with a different histology or with same histology but located in a different segment or lobe. On the contrary, a second tumour is defined as "metachronous" if detected at least 2 years after the primary lesion, with a different or similar histology, but in the second one the cancer must be associated with a premalignant lesion with a different DNA ploidy, and has no systemic metastases or mediastinal spread. Most thoracic surgeons agree that in case of a single lesion in patients who tolerate lung resection well and without distant disease, the newly identified cancer should be resected (5).

Regarding surgical treatment, the type of pulmonary resection depends essentially on the patient's cardiorespiratory function. In patients with synchronous or metachronous tumours located in a different lobe, lobectomy is the treatment of choice. When the patient does not have a good performance status and an adequate cardiopulmonary reserve, it is possible to opt for sub lobar resections, with preference for segmentectomy. This procedure, associated with a systematic lymphadenectomy, results in a survival rate similar to lobectomy when used on tumours of $2 \mathrm{~cm}$ or smaller (6). An aggressive and radical surgical approach was also recommended by other 
authors (7), always considering that patients with limited pulmonary reserve can be treated with limited resection (segmentectomy or wedge resection), or definitive nonoperative local therapy such as stereotactic ablative radiotherapy (SABR) or chemotherapy (8). Lee et al. (9) suggest that in patients with a metachronous lung cancer, an early staging has a big impact on survival after surgical resection. Zuin et al. (10) in their case series of 121 patients explained how a second resection consisting of a completion pneumonectomy had a less favourable 5 -years survival when compared to a lobectomy. According to Verhagen et al. (11), and in contrast with Okada et al. (12), the patients with a metachronous lesion had a better 5 -yearssurvival than the synchronous group. Riquet et al. demonstrated that the 5-years survival rate was increased in patients with synchronous MPLC in one lobe compared with those with synchronous MPLC in another lobe (13).

In any case and as suggested by Rosengart et al. (14) and Jiang et al. (15), patients with synchronous or metachronous MPLC had a higher survival rate when compared with those with metastatic or locally recurrent disease metastasis (patients with synchronous or metachronous MPLC reported to have a $23 \% 5$-year survival rate). In one of their papers Zhang et al. (16) demonstrated that the location of simultaneous tumours did not appear to be related to survival, regardless of bilaterality or location in the same lobe. Similar results had already been described by Voltolini et al. (17), demonstrating that the location of synchronous tumours did not affect survival and that, in the presence of adequate cardiopulmonary function, surgical treatment of lesions is always recommended. Concerning adjuvant chemotherapy, Yu et al. recommends to consider the pathological staging of each tumour separately and to follow the guidelines rather than considering the two lesions as an advanced disease (18).

We describe in our report a 68 -year-old male patient who had undergone left sided VATS basal segmentectomy and lymphadenectomy for SCC (pT2aNOM0R0). He subsequently developed two new primary lung tumours in the left upper lobe within a year. A left VATS (single port) upper trisegmentectomy and lymphadenectomy was performed. Histopathology revealed multiple primary tumours, which were staged separately in lines with what $\mathrm{Yu}$ et al. recommend (18). The surgery achieved R0 resection. Our pathology team agreed that the specimen represented multiple primary tumours when compared with the first tumour excised, arising on a background of field change rather than intrapulmonary metastasis from an index lesion, given the different morphologies of the two subsequent tumours. Vieira $e t$ al. agree with our observation that uniportal VATS approach can easily be used, in the presence of synchronous multiple primary lung cancer (SMPLC), even for a second lobectomy on the side previously operated; despite the presence of adhesions, surgical R0 excision can still be carried out without particular problems (19). The diagnosis of synchronous multiple primary non-small cell lung cancer (SMPNSCLC) had a significant impact on the management protocol as if it was otherwise considered to be stage pT3N0M0, the patient might have needed adjuvant chemotherapy (NCCN guidelines 2019, non-small cell lung cancer).

\section{Conclusions}

MPLC is an up-coming phenomenon in the world of lung cancer and subsequently in thoracic surgery. Encountering such cases can lead to confusion about the management protocol. We wish to add to the literature on MPLC, especially its sub division SMPNSCLC, by describing our patient and our management of offering uniportal VATS surgery to the same side previously operated. We hope that this case report can be a comprehensive read on SMPNSCLC.

\section{Acknowledgments}

Funding: None.

\section{Footnote}

Conflicts of Interest: All authors have completed the ICMJE uniform disclosure form (available at http://dx.doi. org/10.21037/ccts.2019.11.04). The authors have no conflicts of interest to declare.

Ethical Statement: The authors are accountable for all aspects of the work in ensuring that questions related to the accuracy or integrity of any part of the work are appropriately investigated and resolved. Written informed consent was obtained from the patient for publication of this manuscript and any accompanying images.

Open Access Statement: This is an Open Access article distributed in accordance with the Creative Commons Attribution-NonCommercial-NoDerivs 4.0 International License (CC BY-NC-ND 4.0), which permits the non- 
commercial replication and distribution of the article with the strict proviso that no changes or edits are made and the original work is properly cited (including links to both the formal publication through the relevant DOI and the license). See: https://creativecommons.org/licenses/by-nc-nd/4.0/.

\section{References}

1. Johnson BE, Cortazar P, Chute JP. Second lung cancers in patients successfully treated for lung cancer. Semin Oncol 1997;24:492-9.

2. Beyreuther H. Multiplicität von Carcinomen bei einem Fall von sog. "Schneeberger" Lungenkrebs mit Tuberkulose. Virchows Archiv 1924;250:230-43.

3. Martini N, Melamed MR. Multiple primary lung cancers. J Thorac Cardiovasc Surg 1975;70:606-12.

4. Antakli T, Schaefer RF, Rutherford JE, et al. Second primary lung cancer. Ann Thorac Surg 1995;59:863-6.

5. De Leyn P, Moons J, Vansteenkiste J, et al. Survival after resection of synchronous bilateral lung cancers. Eur J Cardiothorac Surg 2008;34:1215-22.

6. Okada M, Yoshikawa K, Hatta T, et al. Is segmentectomy with lymph node assessment an alternative to lobectomy for non-small cell lung cancer of $2 \mathrm{~cm}$ or smaller? Ann Thorac Surg 2001;71:956-60.

7. Chang YL, Wu CT, Lee YC. Surgical treatment of synchronous multiple primary lung cancers: experience of 92 patients. J Thorac Cardiovasc Surg 2007;134:630-7.

8. Oskan F, Kornhuber C, Krause G, et al. Simultaneous stereotactic body radiation therapy of a primary non-small cell lung cancer and synchronous carcinoma in situ in a medically inoperable patient: case report. Radiat Oncol 2013;8:213.

9. Lee BE, Port JL, Stiles BM, et al. TNM stage is the most important determinant of survival in metachronous lung

doi: $10.21037 /$ ccts.2019.11.04

Cite this article as: Guha S, Cascone R, Carlucci A, Kitsanta P, Socci L. Synchronous multiple primary non-small cell lung cancer or intrapulmonary metastasis from an index lesion: a dilemma-case report. Curr Chall Thorac Surg 2019;1:24. cancer. Ann Thorac Surg 2009;88:1100-5.

10. Zuin A, Andriolo LG, Marulli G, et al. Is lobectomy really more effective than sublobar resection in the surgical treatment of second primary lung cancer? Eur J Cardiothorac Surg 2013;44:e120-5; discussion e125.

11. Verhagen AF, Tavila G, van de Wal HJ, et al. Multiple primary lung cancers. Thorac Cardiovasc Surg 1994;42:40-4.

12. Okada M, Tsubota N, Yoshimura M, et al. Operative approach for multiple primary lung carcinomas. J Thorac Cardiovasc Surg 1998;115:836-40.

13. Riquet M, Cazes A, Pfeuty K, et al. Multiple lung cancers prognosis: what about histology? Ann Thorac Surg 2008;86:921-6.

14. Rosengart TK, Martini N, Ghosn P, et al. Multiple primary lung carcinomas: prognosis and treatment. Ann Thorac Surg 1991;52:773-8, discussion 778-9.

15. Jiang L, He J, Shi X, et al. Prognosis of synchronous and metachronous multiple primary lung cancers: systematic review and meta-analysis. Lung Cancer 2015;87:303-10.

16. Zhang Z, Gao S, Mao Y, et al. Surgical Outcomes of Synchronous Multiple Primary Non-Small Cell Lung Cancers. Sci Rep 2016;6:23252.

17. Voltolini L, Rapicetta C, Luzzi L, et al. Surgical treatment of synchronous multiple lung cancer located in a different lobe or lung: high survival in node-negative subgroup. Eur J Cardiothorac Surg 2010;37:1198-204.

18. Yu YC, Hsu PK, Yeh YC, et al. Surgical results of synchronous multiple primary lung cancers: similar to the stage-matched solitary primary lung cancers? Ann Thorac Surg 2013;96:1966-74.

19. Vieira A, Ugalde Figueroa P. Uniportal middle lobectomy after a previous right upper lobectomy. Interact Cardiovasc Thorac Surg 2017;24:477-8. 